\title{
An Assessment of Toxic in Urban Soils Using Garden Cress (Lepidium sativum) in Vasileostrovsky Ostrov and Elagin Ostrov, Saint Petersburg, Russia
}

\author{
Kwabena Awere Gyekye ${ }^{1}$ \\ ${ }^{1}$ Department of Geography and Resource Development, University of Ghana, Accra, Ghana \\ Correspondence: Kwabena Awere Gyekye, Department of Geography and Resource Development, University of \\ Ghana, Legon, Accra, Ghana. Tel: 0547041803. E-mail: kagyekye@ug.edu.gh
}

Received: August 5, 2013 Accepted: October 12, 2013 Online Published: November 8, 2013

doi:10.5539/jgg.v5n4p63 URL: http://dx.doi.org/10.5539/jgg.v5n4p63

\begin{abstract}
The study was conducted to detect toxicity in urban soils of Vasileostrovsky Ostrov and Elagin Ostrov in St. Petersburg, Russia, an area characterised by wide variation in land use. A total of 37 soil samples were collected from the two study areas. Garden cress, Lepidium sativum, $($ L. sativum) was used as the test organism to detect the presence of toxic soils. The results indicated that soils from Vasileostrovsky Ostrov were toxic to L. sativum; the level of toxicity ranged between $40-60 \%$ (mild to moderately toxic). Tests of soils from Elagin Ostrov revealed that soils, generally, were nontoxic $(90-100 \%)$. The results of most examined samples showed that soil extract had a stimulating effect on the growth of fronds; however there were few instances whereby soil extract inhibited the growth of L. sativum.

The results from the study indicated that $L$. sativum is capable of detecting toxic soils. The different reactions from $L$. sativum to soil extracts could be attributed to site-specific conditions.

The study recommends the use of $L$. sativum as a test organism to conduct biomonitoring of urban soil on a wide scale because of its simplicity, sensitivity, and cost-effectiveness.
\end{abstract}

Keywords: bioindication, toxicity, Lepidium sativum

\section{Introduction}

Contaminated soils have come under increased focus for regulators; soils contain a record of the environmental history of an area and can pose a primary environmental risk if not given the necessary appraisal and monitoring attention (Andreiva, Baeva, \& Barkova, 1998; Nautilus Environmental, 2011). Soil toxicity tests are used to provide an accurate and reliable evaluation of the combined toxicity of chemical substances that are bioavailable in the soil (Nautilus Environmental, 2011).

Growing social concern about environmental quality is perceived to be on the increase in recent years, both on a global and local scale. This is connected with more convincing evidence that environmental pollution, for example, results in degradation of particular ecosystems (Gadzała-Kopciuch, Buszewski, Bartoszewicz, \& Buszewski, 2004). Generally, emission of harmful substances from human activities into the natural environments has negative effects on both the environment and human health. It has been observed that chronic toxic effects, impossible to notice at the initial stage of the process, may manifest themselves after many years (Borisenko, 1989; Nałęcz-Jawecki \& Sawicki, 1998; Alloway \& Ayres, 1997; Gadzala-Kopcuich et al., 2004).

In particular, toxic micropollutants which are predominantly the consequence of human activities such as industrialization, urbanisation, and agriculture are the most critical problems concerning environmental protection and urban soil management (Kuprianov, 1977; Vladimirova, Mikulina, \& Yagina, 1986; Kasimov, 1995; Dobrovolsky, 1997). The use of potential bioindicators (plants, animals and/or microbes) as organisms in artificially and historically contaminated soil(s) has proven to be essential in investigation, detection and quantification of toxic activity in the natural environment (Maila \& Cloete, 2002).

Anthropogenic impact on soil quality is often thought of in simple terms, such as discharging wastes from sewage collection systems or industrial outfalls (Dobrovolsky \& Nikitin, 1986). Without doubt, these point sources can have major impacts on receiving soils (Baeva \& Golubeva, 1999). The changes in soil quality which 
are caused by these activities are often cumulative in effect and difficult to remedy because of the widely scattered sources of waste generation. Surface runoff, as complex environmental mixtures, contains an enormous number of potentially polluting substances, a potential source of environmental pollution (Thornton et al., 2001).

In most cases, conventional methods have been used to assess the quality of environmental resources. Apparently, physico-chemical analyses are often insufficient to provide insight into the potential ecological risk, since they do not allow an evaluation of possible combined effects of the different contaminants, as well as to their bioavailability (Maila \& Cloete, 2002). Plant assays are highly sensitive to many environmental pollutants, including heavy metals and have been used to monitor the potential synergistic effects of mixtures of pollutants (Wang \& Freemark, 1995; Nautilus Environmental, 2011).

The present study examines the growth of L. sativum in soils extracts with the aim of detecting soil toxicity and to evaluate its potential as a bioindicator of soil pollution. Soil extracts have been shown to increase or decrease the potential growth weight of L. sativum in soil (Aronstein, Calvillo, \& Alexander, 1991). L. sativum is a fast-growing, edible herb that is botanically related to watercress and mustard, sharing their peppery, tangy flavour and aroma. In some regions, garden cress is known as garden pepper cress, pepper grass, pepperwort or poor man's pepper (Cassidy \& Hall, 2002; Staub \& Buchert, 2008). Lepidium sativum L. (Brassicaceae), commonly known as Chandrasur in Ayurveda is a Rabi crop predominantly grown in temperate regions. Since ancient times, garden cress seeds, leaves, roots and flowers have been regularly used to treat various diseases or disorders (Mukhopadhyay et al., 2010).

Urban health related problems have been correlated with urban pollution, especially with the carcinogenic elements such as heavy metals which are more commonly found in soils (Golubeva \& Sorokina, 2002). These discharged pollutants become dangerous to soils and aquatic life (Singh, Misra, \& Pandey, 2008). With increased urban pollution, serious concerns have been raised about the quality of the urban environment and the necessity for quick remedial action.

A number of researchers (Ufimseva \& Terekhin, 2000) consider that one of the main approaches to assessing the ecological state of the urban environment is by physico-chemical methods. However, it is far from being always true that conclusions made on the basis of physico-chemical results coincide with the bioquality of urban soils to support living organisms.

Meanwhile, the high price and insufficient effectiveness of these techniques have led to the search for more eco-nomical and simple procedures for environmental assessment. In the past few years, investigations have focused on bioindicators that accumulate toxic substances. These investigations gave birth to the technique and application of phytoremediation used in industrial waste-water, which involves the removal of heavy metals by adsorption, accumulation, or precipitation using higher aquatic and terrestrial plants (Gailuilin et al., 2001). These techniques include the wide use of L. sativum as a bioindicator for ecotoxicological assessment of soils (Oleszczuk, 2008), and as a method to evaluate polycyclic aromatic hydrocarbons removal from contaminated soils (Maila \& Cloete, 2002). This can aid the assess-ment of the ecological risk from contaminated soil and also as phytoremediation method in determining the presence of toxic in soils for sustainable monitoring (Maila \& Cloete, 2002).

The available literature on comparing test systems on a rational basis indicates that none of the comparing system sensitivity of different organisms/biotests is more sensitive in all classes of tested toxicants than any other (Eberius, 2001). However, biotest results have proven sensitive and reliable in determining the complex state of an ecosystem and as selected methods of environmental quality assessment (Maila \& Cloete, 2002; Gadzała-Kopciuch et al., 2004).

This study is driven by the fact that the ambiguity of results obtained on ecological state of various sections of urban territories of St. Petersburg, specified the need to conduct further research into urban soils by methods of bioindication, which is widely used in landscape ecology.

The important aspect in adopting bioindication methods in landscape ecology research is the ability to establish a link between an indicator and the physical and chemical characteristics of urban soils as the basis for determining the quality of the urban environment.

The objective of the study was to determine the presence of toxic in soil and to evaluate the sensitivity of $L$. sativum to soil toxicity. 


\section{Materials and Methods}

\subsection{Study Areas}

Two suburbs, Vasileostrovsky Ostrov and Elagin Ostrov in St. Petersburg city of Russia were chosen as sampling sites. The two study areas are fairly close to each other, however, they are quiet distinct in terms of land use. Vasileostrovsky Ostrov, administrative unit of Saint Petersburg is characterized by settlements, educational, social, commercial and industrial activities. Elagin Ostrov, on the other hand, serves as a recreational model zone made up of parks and gardens with limited physical structures. It is situated about $4 \mathrm{~km}$ away from the centre of Vasileostrovsky Ostrov. The two study areas border the delta of the River Neva, at the eastern end of the Gulf of Finland. Most of the city is built on both banks of the Neva, and on islands in the river.

By soil classification, Vasileostrovsky Ostrov and Elagin Ostrov belong to the typical urban soil - courtyard, paths, children's playgrounds; cultivated areas - lawns, parks.

In Vasileostrovsky Ostrov lawns, parks, areas of some few distances away from major roads, and immediate proximity to pedestrian walkways were mapped out and investigated. Some of these sites have been investigated by other researchers using a technique known as "plant-indication" (Terekhin, 1998; Ufimseva \& Terekhin, 2000)

At Elagin Ostrov, soil sampling sites were located at different microenvironment and under varying degree of anthropogenic influence.

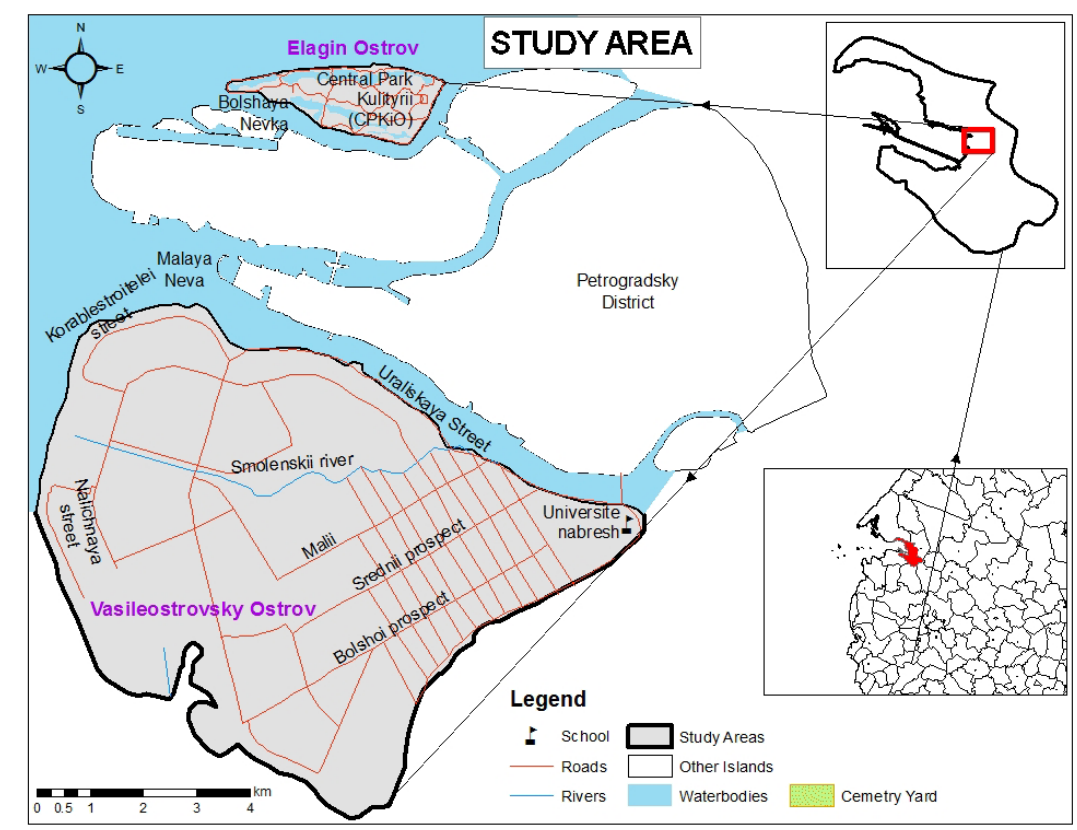

Figure 1. Map showing the study area, Vasileostrovsky Ostrov and Elagin Ostrov

\subsection{Soil Extraction Procedures/Sampling and Experimental Design}

The study examined the growth of L. sativum in soil extracts from Vasileostrovsky Ostrov and Elagin Ostrov. $L$. sativum is widely use in ecotoxicological testing and possesses morphological and physiological properties (small size, high growth rates, and vegetative propagation). Moreover, $L$. sativum grows in a wide range of $\mathrm{pH}$-values ( $\mathrm{pH} 3.5-10)$ which makes it an ideal organism for toxicity testing of pollutants in waste waters (Wang \& Freemark, 1995).

Before extraction, the dry soil samples were screened to a fineness of $0.2 \mathrm{~mm}$ using the Hach Soil Sieve in the kit. This was followed by dissolving $5 \mathrm{~g}$ of soil in $25 \mathrm{ml}$ distilled water in a $50 \mathrm{ml}$ plastic beaker and the solution was filtered to obtain the extract for the biotest analysis. Next, dry seeds of L. sativum were germinated in pre-sterilized petri dishes lined with a two-layer filter paper and soaked with $10 \mathrm{ml}$ soil extract of same concentration. Distilled water and non contaminated soil was used for the control experiment. Each petri dish contained 50 dry seeds of $L$. sativum. All samples and their controls were replicated. The petri dishes were 
placed next to a window allowing sufficient light for photosynthesis. After every 48 hours, $5 \mathrm{ml}$ of soil extract was evenly added onto the filter paper during the 7 days growth period.

A growth measure of $L$. sativum was carried out on the 7th day of growth. Fresh weight fronds were collected from each petri dish and blotted to remove excess water and dried for 24 hours at $50^{\circ} \mathrm{C}$ in a laboratory micro oven dryer mainly consists of magnetron tube (source of radiation), oven cavity, filter, step-up transformer, power plug, wave guide, mode stirrer and oven tray. The absolute dry weight of each germinated sample was weighed (to $0.001 \mathrm{~g}$ ) on an analytical balance. In all 31 samples were analysed: 20 samples were taken from Vasileostrovsky Ostrov and 11 from Elagin Ostrov.

\subsection{Analysis}

Toxicity was calculated on the 7th day of growth for the controls and for each sample group. The method for computation is as follows:

$$
\mathrm{T}=\mathrm{AvDrWt} / \mathrm{AvCDrWt} \mathrm{X} 100 \%
$$

Where,

\section{$\mathrm{T}=$ Toxicity, AvDrWt (Average Dry Weight), AvCDrWt (Average Control Dry Weight)}

The indicators used to assess the toxicity of soils were based on absolute fresh and dry weights of harvested fronds; these indicators were found to be more acceptable because some substances may affect the frond size without affecting the frond number or vice-visa. The results for each replicate and control were averaged and assessed statistically for mean of 62 samples ( 2 replicates x 31 samples per 50 seeds per sample). The statistical significance level in this study was defined at $\mathrm{P}<0.05$. The statistical processing was performed with SPSS for Windows release 17.0.

Toxicity scaling: The index of toxicity $(\mathrm{T})$ was applied to represent the degree of toxicity of soil and the results were determined on the basis of measurement of dry and fresh weight sprouts of $L$. sativum (Fedorov \& Nikolskaya, 1997) (Table 1).

Table1. Scale of soil toxicity for urban soils (Fedorov \& Nikolskaya, 1997)

\begin{tabular}{cc}
\hline Index of soil Toxicity $(\mathrm{T}) \%$. & Interpretation of Index, Toxicity $(\mathrm{T})$ of soil \\
\hline $100-90$ & Nontoxic \\
$80-90$ & Mildly toxic \\
$60-80$ & Moderately toxic \\
$60-40$ & Toxic \\
$40-20$ & Strongly toxic \\
$0-20$ & Lethally toxic \\
\hline
\end{tabular}

\section{Results and Discussions}

\subsection{Weight Gain and Loss of Sprouts}

The current study assesses the potential of L. sativum to detect toxic soils. The overall potential effect of each soil extract from various land uses on L. sativum was noted. There was significant response of soil extracts to stimulate the growth of L. sativum and subsequently on its biomass. It was noted that soil extract, to a large or small extent, affected the weight of the sprout since there was remarkable difference between the weight of control values and the samples values (Tables 2 and 3). For example, it was observed that the average control weight of fresh sprouts was $1.29 \pm 0.06 \mathrm{~g}$ ( \pm standard error) while that for sprouts germinated in soil extract was $1.59 \pm 0.085(\mathrm{p}<0.05)$, as indicated in Table (2) for Vasileostrovsky Ostrov. Further analysis of weight of sprouts from Vasileostrovsky Ostrov showed that $73.7 \%$ sprouts germinated in soil extracts had weights greater than the average control weight of fresh sprouts, an indication of a possible stimulation effect from soil extracts. A similar situation was observed for L. Sativum growth in soil extracts from Elagin Ostrov for both fresh harvested fronds and dry fronds where average weights of control values were found to be slightly lower than some fronds germinated in soil extract. However, in some limited cases, a contrary situation was noted where control values were found to be slightly higher than observed values (Table 3 ) giving the impression that soil extracts inhibited the growth of fronds and, subsequently, led to weight loss of biomass. 
In an attempt to assess the possible influence of site conditions on $L$ sativum (in this regard total concentration of heavy metals in soils) a superimposed analysis was carried out. This analysis compared the total concentration of heavy metals in soil from sites used in the preparation of soil extract for the growth of $L$. sativum with soil from sites in the investigated areas. This was considered to be essential since the concentration of heavy metals in most urban soils, on the average, exceeded the acceptable concentration limit by $100 \mathrm{mg} / \mathrm{kg}$ soil and this may have far-reaching implications on soil quality (Grigoryan \& Saet, 1980). The result of the comparison indicated that areas having sprouts weight greater than the average control value fell within sampled areas where the index of total concentration of heavy metals in soils ( $\mathrm{ZcHM}$ ) ranged within 32-128 classified as dangerous, and 128-132, extremely dangerous (Andreiva et al., 1998). Heavy metals constitute one of the most dangerous pollutants of the environment and research findings have indicated that increasing concentration of heavy metals above permissible levels most often lead to inhibition of the functional activities of living organisms and subsequently degradation of the ecosystem (Dobrovolsky, 1997). Today it is well known that a number of toxicants (for example, salts of heavy metals) in small concentration strengthen growth processes, and in high concentration act as inhibitors to growth (Fedorov \& Nikolskaya, 1997). From this analysis, it can be said that the biological response to soils samples appears to be related to the chemical characteristics of soils in the study area.

The use of biotest to assess the state of urban soils takes into consideration that territorial heterogeneity is an important ecological factor (variation in moisture, temperature, light exposure, etc.) which can influence research results. The test - object used in the research detected the presence of chemical limiting factors in soils. This is consistent with the findings of other research (Fedorov \& Nikolskaya, 1997).

Based on the scaling of toxicity, the results indicated the presence of toxicity in urban soils in western, central, and eastern parts of Vasileostrovsky Ostrov. However, the results revealed significant variation in the level of toxicity of sampled soils ranging from nontoxic, moderately toxic, to toxic (Figure 2). Results from Elagin Ostrov indicated that about $50 \%$ of tested soils are nontoxic ranging from 90 to $100 \% ; 40 \%$ of tested soils show weak toxicity, $80-90 \%$, and only $10 \%$ of tested soils are moderately toxic, $60-80 \%$ (Figure 3 ).

The statistical analysis of sprouts of $L$. sativum from the two study areas showed insignificant coefficient of variation as indicated in Table 2, which suggests that there is inconsequential difference in the level of toxicity from the observed values. However, it can be said that although the degree of variation is insignificant, it is the indicator's response to the soil extract that is significant and which also indicates that there is a link between the indicator and the quality of urban soils.

Table 2. Weight characteristics of sprouts of $L$. sativum from Vasileostrovsky Ostrov

\begin{tabular}{ccccccc}
\hline & Min & Max & Mean & Error & Std. SD & CV \\
\hline Fresh weight & 1.04 & 2.18 & 1.5947 & .08534 & .37198 & .138 \\
Fresh weight Control & 1.23 & 1.35 & 1.2900 & .06000 & .08485 & .007 \\
Dry weight & .04 & .12 & .0818 & .00467 & .02036 & .000 \\
Dry weight Control & .05 & .07 & .0600 & .01000 & .01414 & .000 \\
\hline
\end{tabular}

Table 3. Weight characteristics of sprouts of L. sativum from Elagin Ostrov

\begin{tabular}{ccccccc}
\hline & Min & Max & Mean & Error & Std.SD & CV \\
\hline Fresh weight & 1.10 & 1.84 & 1.5560 & .08232 & .26031 & .068 \\
Fresh weight (Control) & 1.75 & 1.87 & 1.8100 & .06000 & .08485 & .007 \\
Dry weight & .06 & .10 & .0860 & .00356 & .01125 & .000 \\
Dry weight (control) & .10 & .10 & .1000 & .00000 & .00000 & .000 \\
\hline
\end{tabular}




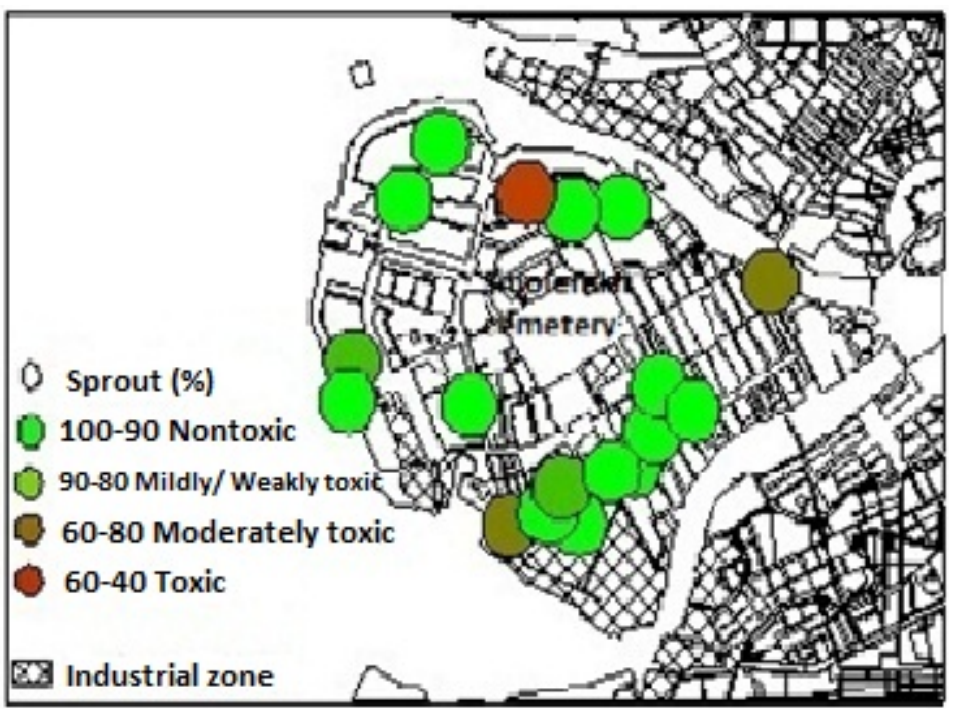

Figure 2. Map showing the level of toxicity by L.sativum in Vasileostrovsky Ostrov

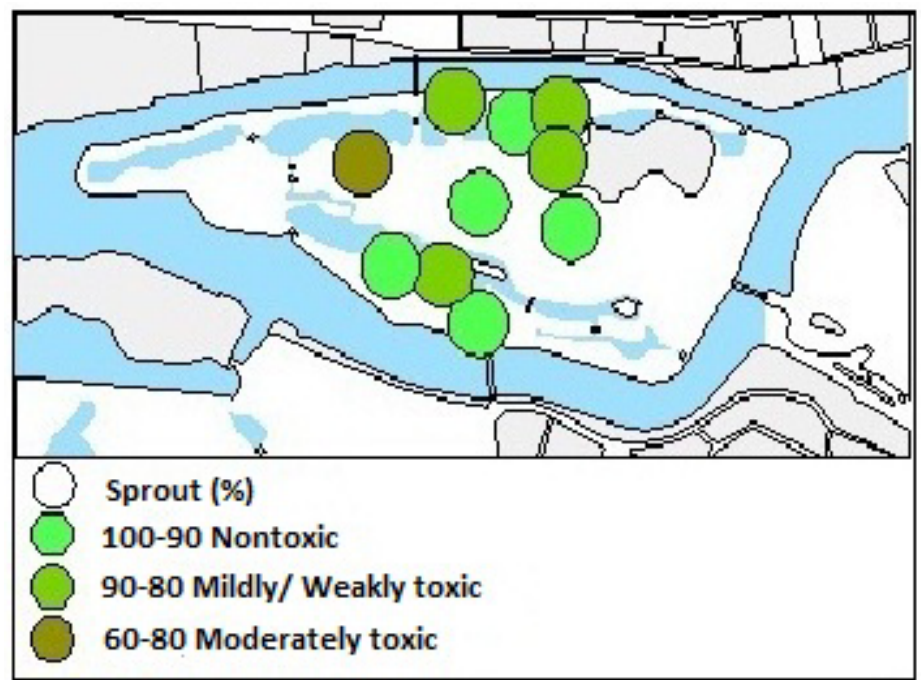

Figure 3. Map showing the level of toxicity by Lepidium sativum in Elagin Ostrov

\section{Conclusions}

Lepidium sativum was grown in soil extract to determine the presence of soil toxicity. The dry weight of $L$. sativum was used as the basis for measuring the level toxicity. Analysis of the results showed that the level of toxicity varied from non-toxic, weakly toxic, to moderately toxic soils in Vasileostrovsky Ostrov which might have been influenced by territorial heterogeneity of urban soils on the test object, L. Sativum. Most soils in Elagin Ostrov were found to be nontoxic, only one soil sample was found to be moderately toxic.

From the point of view of land use type and it influence on the quality of urban soils, it can be argued that soils in Elagin Ostrov are more favourable for L. sativum as a living organism than soils in Vasileostrovsky Ostrov. The former is most likely the consequence of insignificant human impacts on the environment and effects of landscape gardening services carried out there.

The variations in the level of soil toxicity suggest that the heterogeneous nature of urban soils might have influenced the sensitivity of the test organism, L. sativum to impacts. Therefore, it can be concluded that $L$. sativum reaction to urban soils of Vasileostrovsky Ostrov and Elagin Ostrov is not as much the result of human impact as it is the result of complex ecological factors. These ecological factors include spatial variability of soil properties which might have influenced the indicator's tolerance and reaction to soil extracts. 
The study revealed that soil extract stimulated as well as inhibited the growth of $L$. sativum. This was indicated by the fact that the weight of fresh and dry fronds of $L$. sativum varied from the control values.

Results of this study revealed that differences in the level of soil toxicity were insignificant as indicated by the low coefficient of variation for the analysed values. The following factors may account for this: (a) the highly homogeneous plant nature of L. sativum assay, (b) its near-uniform weight distribution, and (c) the homogeneity of genetic make-up.

The study recommends the use of $L$. sativum as a monitoring tool for early detection of contaminated soils in urban agglomeration environments due to its sensitivity to toxicity in soils. Further, the methodology should be used to complement existing traditional monitoring tools to identify specific constituents responsible for toxicity. These methods can, therefore, help to implement more effective remediation treatments.

\section{References}

Alloway, B. J., \& Ayres, D. C. (1997). Chemical Principles of Environmental Pollution. London: Blackie Academic \& Professional.

Andreiva, V. G., Baeva, A. S., \& Barkova, N. V. (1998). Protection of the Environment, Natural Resource Utilization and Providing Ecological Safety of Saint Petersburg in 1998 (p. 442). St. Petersburg.

Aronstein, B. N., Calvillo, Y. M., \& Alexander, M. (1991). Effect of Surfactant at LowConcentrations on the Desorption and Biodegradation of Sorbed Aromatic Compounds in Soil. Environmental Science Technology, 25(10), 1728-1731. http://dx.doi.org/10.1021/es00022a008

Baeva, A. S., \& Golubeva, N. D. (1999). Protection of the Environment, Natural Resource Utilization and Providing Ecological Safety of Saint Petersburg in 1999 (p. 543). St. Petersburg.

Borisenko, I. L. (1989). Technogenic Pollution of Various Hierarchy of Landscape under the Influence of Nonferrous Metallurgical Activity. Biogeochemical Methods of Studying the Environment, Moscow, 4-10.

Cassidy, F. G., \& Hall, J. H. (2002). Dictionary of American Regional English: P-Sk. USA: Belknap Press of Harvard University Press.

Dobrovolsky, G. V. (1997). Soils, Cities, Ecology. Moscow: Science Publishers.

Dobrovolsky, G. V., \& Nikitin, E. D. (1986). Ecological Function of Soils: Test Book for University Students and Specialists in Agrochemistry and Soils. Moscow State University, Moscow.

Eberius, M. (2001). Using the Duckweed Growth Inhibition Test to Detect and Evaluate Soil Contamination.

Fedorov, A. I., \& Nikolskaya, A. N. (1997). Practical in Ecology and Protection of the Environment. Prescribed Book, Voronezh 1997, p. 189

Gadzała-Kopciuch. R., Berecka, B., Bartoszewicz, J., \& Buszewski, B. (2004). Some Considerations about Bioindicators in Environmental Monitoring. Polish Journal of Environmental Studies, 13(5), 453.

Gailuilin, V. G. Rauf., Vladimir N. Bashkin., Rumiya R. Gailuilina., \& Paul Birch. (2001). A Critical Review Protection from Pollution by Heavy Metal-Phytoremediation of Industrial Waste Water. Land Contamination and Reclamation, 9(4).

Grigoryan, S. V. \& Saet J. E. (1980). Geochemical Methods of Assessing Some Ecological Problems (p. 11). The Soviet Geology.

Kasimov, N. S (1995). Ecogeochemistry of Urban Landscapes. Moscow: Publishing House of Moscow State University.

Kuprianov, V. V. (1997). Hydrological Aspects of Urban Environment of Leningrad. Leningrad: Hydro Publication.

Maila, M. P., \& Cloete, T. E. (2002). Germination of Lepidium Sativum as a Method to Evaluate Polycyclic Aromatic Hydrocarbons (PAHs) Removal from Contaminated Soil. International Biodeterioration and Biodegradation, 50(2), 107-113. http://dx.doi.org/10.1016/S0964-8305(02)00059-8

Mukhopadhyay, D., Parihar, S. S., Chauhan, J. S., \& Preeti, S. C. J. (2010). Effect of Temperature and Desiccation on Seed Viability of Lepidium sativum L. New York Science Journal, 3(5).

Nałęcz-Jawecki, G., \& Sawicki, J. (1998). Toxicity of Inorganic Compounds in the Spirotox Test: A Miniaturized Version of the Spirostomum Ambiguum Test. Arch Environ Contam Toxicol, 34(1), 1-5. http://dx.doi.org/10.1007/s002449900278 
Nautilus Environmental. (2011). Soil Toxicity: Environmental services. San Diego: Nautilus Environmental.

Oleszczuk, P. (2008). Phytotoxicity of municipal sewage sludge composts related to physic-chemical properties of, PAHs and heavy metals. Ecotoxicology and Environmental Safety, 69(3), 496-505. http://dx.doi.org/10.1016/j.ecoenv.2007.04.006

Singh, K., Misra, A., \& Pandey, S. N. (2008). Responses of Lemna minor L.(duckweed) plants to the pollutants in industrial waste water. Research in Environment and Life Sciences, 1, 5-8.

Staub, J., \& Buchert, E. (2008). 75 Exceptional Herbs for Your Garden. Layton: Gibbs Smith.

Terekhin, N. B. (1998). Multi-criteria Method of Phytoindication Assessment of Ecological State of Urban Megapolis Environment: for example Vasileostrovsky Ostrov Area of Saint Petersburg (Doctoral dissertation). Saint Petersburg State University, Saint Petersburg.

Thornton, I., Butler, D., Docx, P., Hession, M., Makropoulos, C., McMullen, M., ... White, D. (2001). Pollutants in Urban Waste Water and Waste Sludge. Final Report Prepared by ICON IC Consultants Ltd. Luxemburg: European Commission.

Ufimseva, M. D., \& Terekhin, N. B. (2000). Express Phytoindication Method of Assessing Ecological State of the Environment. Methodical Directives, Saint Petersburg: Petersburg State University.

Vladimirova, V. V., Мiкulina, Е. M., \& Yagina, Z. N. (1986). Town and Landshaft. Moscow Misili, p 238.

Wang, W., \& Freemark, K. (1995). The use of plants for environmental monitoring and assessment, Ecotoxicology and Environmental Safety, 30(3), 289-301. http://dx.doi.org/10.1006/eesa.1995.1033

\section{Copyrights}

Copyright for this article is retained by the author(s), with first publication rights granted to the journal.

This is an open-access article distributed under the terms and conditions of the Creative Commons Attribution license (http://creativecommons.org/licenses/by/3.0/). 